\title{
Impact of Solubility Enhancement Methods on the Dissolution Rate of Valsartan Sachet
}

\author{
Anjam H. Abdalla, Anoosh B. Hagop*, Dina A. Boya \\ Department of Pharmaceutics, College of Pharmacy, Hawler Medical University, Erbil, Kurdistan Region, Iraq
}

\author{
${ }^{*}$ Corresponding author: \\ Anoosh B. Hagop, \\ Department of \\ Pharmaceutics, College of \\ Pharmacy, Hawler Medical \\ University, Erbil, Kurdistan \\ Region, Iraq. \\ E-mail: anush.hakub@hmu. \\ edu.krd
}

Received: 23 September 2020

Accepted: 17 February 2021

Published: 30 June 2021

DOI

10.25156/ptj.v11n1y2021.pp63-69

\section{A B S T R A C T}

The oral drug delivery is the most generally used route of administration that has been explored for the delivery of drugs through various pharmaceutical products. Solubility of drug plays critical role in achieving the optimum therapeutic levels of the drug in blood and thus bioavailability. There are many drugs of various therapeutic categories fall in Biopharmaceutics Classification System Classes II and IV as they lack solubility. For all these drugs, dissolution is the big issue for the absorption process. Valsartan is an effective antihypertensive agent and it can be used for the treatment of hypertension in most cases. The objective of this study is to prepare Valsartan as an oral sachet which can be used as an alternative dosage form after improvement of drug solubility using solubilizing agents such as sodium lauryl sulfate and tween 80 . Three different formulas of Valsartan sachet were prepared by conventional technique of wet granulation method named conventional formula ( $\mathrm{Fc}$ ), sodium lauryl sulfate formula ( $\mathrm{Fs}$ ), and tween 80 formula (Ft) then compared with the available marketed product of Valsartan tablet (Fd) as a reference. The preformulations studies were conducted to exclude drug excipients interaction. Evaluation was performed in terms of weight variation, dose content uniformity, and drug release study using dissolution test apparatus. Fourier Transforms Infrared Spectroscopy reveals no drug excipient interaction and the drug release profile for Fs and $\mathrm{Ft}$ formulas within 30 min was $100.16 \%$ and $104.16 \%$, respectively, while for Fc only $57.55 \%$ of the drug was released. This difference in the release profile was statistically significant $(P<0.05)$ between $\mathrm{Fs}$ and $\mathrm{Ft}$ with $\mathrm{Fc}$, but a non- significant difference $(P>0.05)$ was observed between $\mathrm{Fs}$ and $\mathrm{Ft}$ with the marketed Valsartan tablet (Fd). The results support the possibility of using the prepared formulas Fs and $\mathrm{Ft}$ as a Valsartan sachet for the oral administration alternative to conventional Valsartan tablets Fd.

Keyword: Poor oral solubility; Wet granulation method; Valsartan; Sachet

\section{INTRODUCTION}

Solubility is a property of substance that enables to dissolve in a solvent. Quantitatively solubility referring to the concentration of the solute in a saturated solution at a certain temperature (Bharti et al., 2015). Low aqueous solubility of drug is encounted the rate limiting step for drug absorption after oral administration. Approximately $40 \%$ of marketed drugs and about $90 \%$ of drug molecules in the development pipeline show inadequate water solubility. Hence, solubility is considered the main problem in the pharmaceutical industry while developing a new dosage form. The rate of drug dissolution determines the rate and extent of drug absorption (Rong, 2008),

The soluble part of drug enables the penetration through gastro intestinal membrane while the insoluble part remains within gastro intestinal tract and excretes with feces (Aulton, 2000; Rabasco et al., 2002). Enhancement in drug solubility improves drug absorption, and then it has an effect in minimizing dosing quantity, patient compliance, and cost of drugs (Rabasco et al., 2002).
Various methods are used for the solubility enhancement of poorly water-soluble drugs either physical and/or chemical modification. The solubility of drug molecules intrinsically related to the reduction of particle size, the addition of solubilizing agent, preparing as a solid dispersion, $\mathrm{pH}$ adjustment, and application of complexation (Lobenberg and Amidon, 2000).

According to Biopharmaceutics Classification System (BCS), drugs are classified in to four groups based on the ability of a given drug substance to permeate through biological membranes and aqueous solubility. Drug substances are considered highly soluble when the largest dose of a compound is soluble in $<250 \mathrm{~mL}$ of water over a range of $\mathrm{pH}$ from 1.0 to 7.5 , highly permeable compounds are classified as those compounds that demonstrate $>$ more than $90 \%$ absorption of the administered dose. In contrast, compounds with solubility's below $0.1 \mathrm{mg} / \mathrm{mL}$ face to significant solubilization obstacles, and often even compounds with solubility's below $10 \mathrm{mg} / \mathrm{mL}$ present difficulties related to solubilization during formulation (Pedersen et al., 2000). 
According to BCS system, drugs are classified into four groups as follows: (Kawabataa et al., 2011)

- Class I consists of highly water-soluble and permeable drug.

- Class II consists of water-insoluble with a high permeable drug.

- Class III consists of water-soluble with a low permeable drug.

- Class IV consists of water-insoluble with a low permeable drug.

Valsartan belongs to Class II drug according to BCS system. Valsartan is an angiotensin II-receptor antagonist that has been available overseas for several years which can be used to decrease blood pressure. Valsartan is an effective antihypertensive drug and could decrease blood pressure with less side effects compared to the other antihypertensive agents (Gilman et al., 1990). Valsartan is used in the treatment of pediatric, adolescents, and elderly patients with mild-to-moderate hypertension (Flesch et al., 1997).

Surfactants are commonly used to reduce surface tension between aqueous medium and drug molecules, causing enhancement in drug solubility in the aqueous medium. Surface active agent molecules have a distinct polar and nonpolar regions. The polar group can be anionic, cationic, zwitterionic (amphoteric), or nonionic (Dalmora et al., 2011). The presence of surfactants may lower the surface tension and increase the solubility of the drug within an organic or an aqueous solvent (Lawrence and Rees, 2000).

An ionic surfactants like sodium lauryl sulfate (SLS) are widely employed in the preparation of nonparenteral pharmaceutical formulations and cosmetics. Polyoxyethylene sorbitan fatty acid esters (polysorbates) or what is called tween 80 are a series of partial fatty acid esters of sorbitol. Tween 80 has a characteristic odor and a warm, somewhat bitter taste. It is used as solubilizing agent for enhancing solubility of poorly soluble active constituents in lipophilic bases during preparation of cosmetics and food products (Rowe et al., 2009).

The aim of this study is to prepare Valsartan as a sachet to improve the solubility of Valsartan in aqueous comparing to conventional tablet dosage form. Conventional technique was used to prepare wet granules loaded Valsartan with or without binding agents and surfactants.

\section{MATERIALS AND METHODS}

\section{Materials}

Valsartan was supplied by Awamedica Pharmaceutical Company as a gift. Polyvinyl pyrollidone, SLS, tween 80, lactose, orange flavor, and sorbitol were used as additives and they were supplied by Pharmaceutics Department at College of Pharmacy, Hawler Medical University. Diovan ${ }^{\mathrm{R}}$ was used as a brand for the Valsartan conventional tablet.

\section{Method \\ Melting point determination}

Capillary tube method was used for the measurements of melting point of Valsartan. A tiny amount of Valsartan powder was introduced in to a small glass capillary tube, which is sealed at one end, and placed into melting point apparatus. The sample was heated slowly and carefully observed. The temperature at which melting begins and complete was recorded as a melting point (USP, 2007).

\section{Determination of calibration curve of Valsartan (Pradhan and Mishra, 2015):}

Standard drug solution of Valsartan was prepared by dissolving $10 \mathrm{mg}$ of pure Valsartan in methanol and distilled water (D.W) using beaker. The solution was transferred into $100 \mathrm{ml}$ volumetric flask to obtain $100 \mu \mathrm{g} /$ $\mathrm{ml}$ of stock solution. Standard stock solution was suitably diluted with D.W to obtain a series of concentrations ranging from 5 to $35 \mu \mathrm{g} / \mathrm{ml}$. Absorbance was measured at $250 \mathrm{~nm}\left(\lambda_{\text {max }}\right.$ of Valsartan) using Ultraviolet (UV)Visible spectrophotometer (analytiKjena, SPECORD 40). Calibration curve was drawn by plotting graph between concentration and absorbance.

\section{Drug-excipient compatibility study}

The Fourier Transforms Infrared Spectroscopy (FTIR) (Jasco, FT/IR-4600) was used for the IR analyses in the frequency range between 4000 and $400 \mathrm{~cm}^{-1}$ at $1 \mathrm{~cm}^{-1}$ resolution. The instrument was calibrated using polystyrene film. Pure drug and excipients were separately used (Usha et al., 2015). The IR peak of pure Valsartan was analyzed and compared with the peaks of the prepared formulations (Fc, Fs, and Ft).

\section{Preparation of Valsartan Sachet}

Various formulas were prepared using conventional technique of wet granulation method. The contents of conventional formula $(\mathrm{Fc})$, formulation containing SLS $(\mathrm{Fs})$, and formulations containing tween $80(\mathrm{Ft})$ are shown in Table 1.

All the formulas were prepared by dissolving polyvinyl pyrrolidone in small quantity of D.W then the formed liquid mixture was added to the remaining mixed dry ingredients. The formulas were passed through mesh \# 60 to get wet granules. The wet granules were dried in the oven for $30 \mathrm{~min}$ and the dried granules containing Valsartan prepared as a sachet. 
Table 1: Formulation of Valsartan sachet $\mathbf{3 0 0} \mathbf{~ m g}$

\begin{tabular}{lcccccccc}
\hline Formula name & Valsartan & Polyvinyl pyrrolidone & Orange flavor & Sorbitol & SLS & Tween 80 & Lactose & Total (mg) \\
Fc & 40 & 2.5 & 5 & 20 & 0 & 0 & 232.5 & 300 \\
Fs & 40 & 2.5 & 5 & 20 & 1.5 & 0 & 231 & 300 \\
Ft & 40 & 2.5 & 5 & 20 & 0 & 14 & 218.5 & 300 \\
\hline
\end{tabular}

\section{Evaluation of the Prepared Valsartan Sachet (Nayak et al., 2018)}

1. Weight variation test: Ten sachets were taken and their weights were measured individually and collectively using a digital weighing balance. The average weight of sachets was calculated.

2. Weight content uniformity: Six sachets of $300 \mathrm{mg}$ of Valsartan as dry granules were accurately weighed and transferred into a $100 \mathrm{ml}$ volumetric flask containing a solution of $(10 \mathrm{ml}$ ethanol and $90 \mathrm{ml} \mathrm{D.W})$. The solution was shaken for 10 min using magnetic stirrer, then the solution was filtered, diluted suitably and drug content were quantified using UV-Visible spectrophotometer at $\lambda_{\text {max }} 250 \mathrm{~nm}$.

3. Dissolution test: Release of Valsartan from prepared granules was determined using USP dissolution testing apparatus type II (paddle method). The dissolution test was performed using $900 \mathrm{ml}$ of phosphate buffer solution ( $\mathrm{pH} \mathrm{6.8)}$ at $37 \pm 0.1^{\circ} \mathrm{C}$ and $50 \mathrm{rpm}$. Sample of the solution $(5 \mathrm{ml})$ was withdrawn from the dissolution apparatus at different time intervals and was replaced with fresh dissolution medium. The samples were filtered through a $0.45 \mu$ membrane filter and diluted with phosphate buffer solution ( $\mathrm{pH}$ 6.8). For the filtrated solution, absorbance was taken at $\lambda$ max $250 \mathrm{~nm}$ using a UV-Visible spectrophotometer. Cumulative percentage drug release was calculated using regression equation obtained from a standard calibration curve. Finally, the release characteristics of Valsartan dry granules ( $\mathrm{Fc}$, Fs and $\mathrm{Ft}$ ) were compared with the available marketed drug product of Valsartan tablet (Diovan) ${ }^{\mathrm{R}}$.

\section{Statistical Analysis}

All experiments were carried out in triplicate. The values were represented as mean \pm standard deviation. Evaluation parameters were tested for significance difference using independent two samples $t$-test with the aid of the statistical package for the social sciences (version 18) program. Differences were considered statistically significant when $P<0.05$.

\section{RESULTS}

\section{Melting Point Determination}

The measured melting point reveals a rational purity of Valsartan powder at $117^{\circ} \mathrm{C}$. The acceptable range of Valsartan melting point is $115-120^{\circ} \mathrm{C}$ (Moffat, 2011).
Determination of $\lambda_{\text {max }}$ (Maximum Absorbance)

The prepared solution of Valsartan was scanned by UVVisible spectrophotometer between a wave length of $200-400 \mathrm{~nm}$ using $1 \mathrm{~cm}$ quartz cell. A well-defined $\lambda_{\text {max }}$ at $250 \mathrm{~nm}$ was appeared in the spectrum (Khan et al., 2010), as shown in Figure 1.

\section{Determination of Calibration Curve of Valsartan}

Calibration curve of Valsartan in methanol and distilled water was determined using UV-Visible spectrophotometer at $250 \mathrm{~nm}$. The absorbance was plotted against concentration and calibration curve was drawn. The calibration curve was linear in the concentration range from 5 to $35 \mu \mathrm{g} / \mathrm{ml}$. The correlation coefficient was found to be 0.999 . The calibration plot is illustrated in Figure 2.

\section{Drug-excipient Compatibility Study}

The FTIR was used to evaluate the compatibility of Valsartan with pharmaceutical excipients of common use as diluents and binder. The IR spectra of pure Valsartan drug powder and the prepared formulas (Fc, Fs, and Ft) were taken for the characterization studies, as shown in Figures 3-6, respectively. IR spectrum of pure Valsartan powder showed characteristic peaks at $2963.09 \mathrm{~cm}^{-1}$ due to $\mathrm{C}=\mathrm{N}$ stretching, at $1731.76 \mathrm{~cm}^{-1}$ due to carboxylate stretching. The spectra also showed bands at $1731.76 \mathrm{~cm}^{-1}$ due to $\mathrm{C}=\mathrm{O}$ bending and at $1105.01 \mathrm{~cm}^{-1}$ due to $\mathrm{C}-\mathrm{N}$ bonding (Balamuralidhara et al., 2009).

\section{Evaluation of the Prepared Valsartan Sachet}

1. Weight variation test: The result shows that there was a non-significant differences $(P>0.05)$ between Fc, $\mathrm{Fs}, \mathrm{Ft}$, and $\mathrm{Fd}$ and the results are expressed as a mean \pm S.D $(n=10)$, as shown in Table 2 .

2. Weight content uniformity: The percentage of weight content uniformity of the prepared formulas Fc, Fs, $\mathrm{Ft}$, and $\mathrm{Fd}$ was calculated and listed in Table 3. There was a non-significant differences $(P>0.05)$ between the prepared Valsartan sachet and $\mathrm{Fd}$ and the results are expressed as a mean \pm S.D $(n=6)$.

3. Dissolution test of Fc, Fs, and Ft in comparison with Fd: The dissolution for the formula Fc, Fs, and Ft and Valsartan conventional tablet Fd (Diovan ${ }^{\circledR} 80 \mathrm{mg}$ tablet) as a reference was performed according to USP, using $900 \mathrm{ml}$ of phosphate buffer solution ( $\mathrm{pH}$ 6.8) as a dissolution medium at $37^{\circ} \mathrm{C}$ with constant stirring speed of $50 \mathrm{rpm}$ for $45 \mathrm{~min}$. The results listed in Table 4 and are expressed as a mean \pm S.D $(n=6)$. 


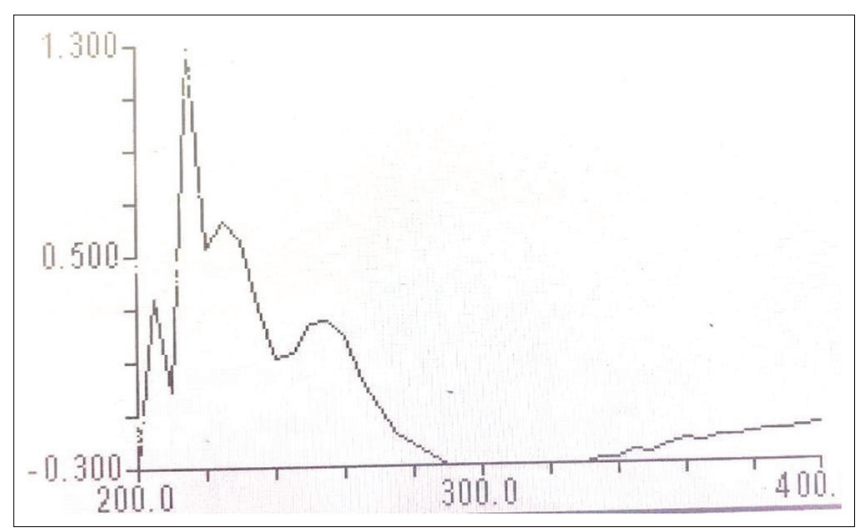

Figure 1: Ultraviolet-scan of Valsartan in a mixture solvent of methanol and phosphate buffer (pH 6.8)

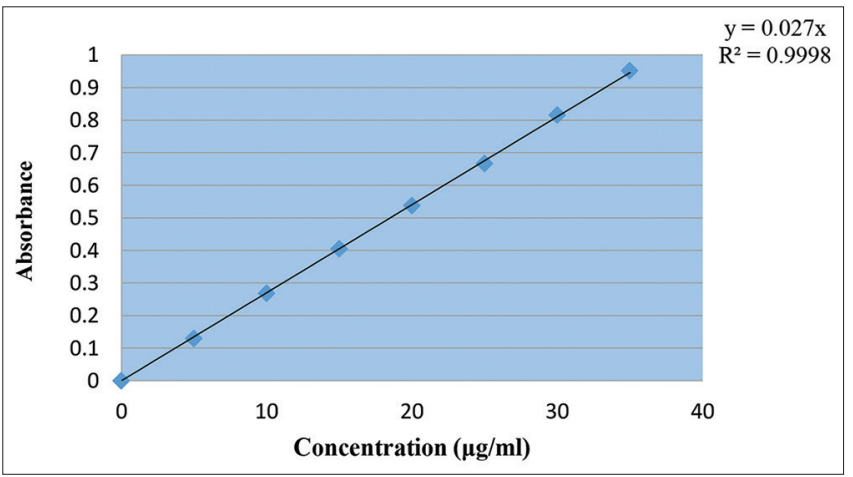

Figure 2: Calibration curve of Valsartan in a mixture solvent of methanol and phosphate buffer (pH 6.8)

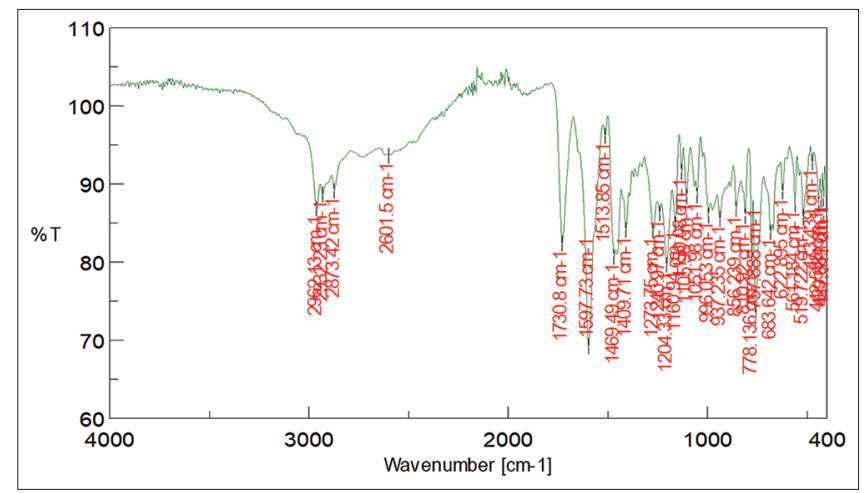

Figure 3: Fourier-transform infrared spectrum of pure Valsartan powder

Figure 7 shows the comparison of the drug release profile of the prepared Valsartan sachet ( $\mathrm{Fc}, \mathrm{Fs}$, and $\mathrm{Ft}$ ) with Valsartan conventional tablet $(\mathrm{Fd})$ as a reference. Depending on the USP for the solid dosage forms $30 \mathrm{~min}$ is needed to release $75 \%$ of the drug content (USP-30). The results showed within $30 \mathrm{~min}$ about $100.16 \%, 104.16 \%$, and $85.95 \%$ of drug were released from Fs, Ft, and Fd, respectively. The results are within the acceptable limit range, while $\mathrm{Fc}$ formula provides $57.55 \%$ drug release.

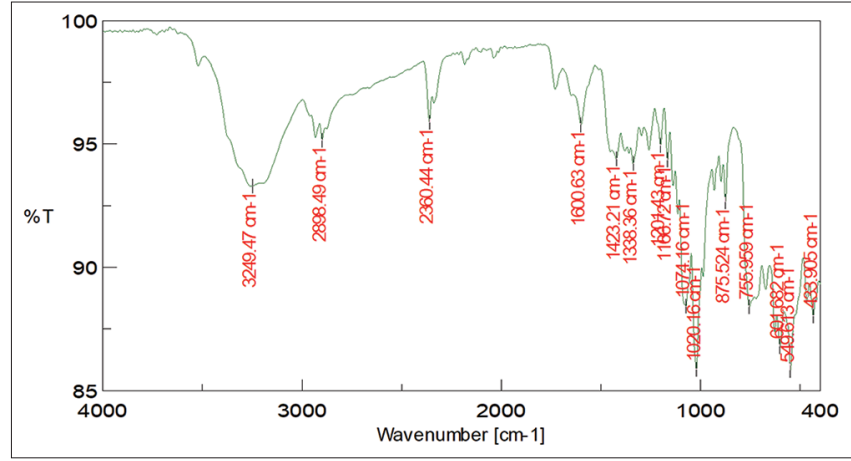

Figure 4: Fourier-transform infrared spectrum of Fc

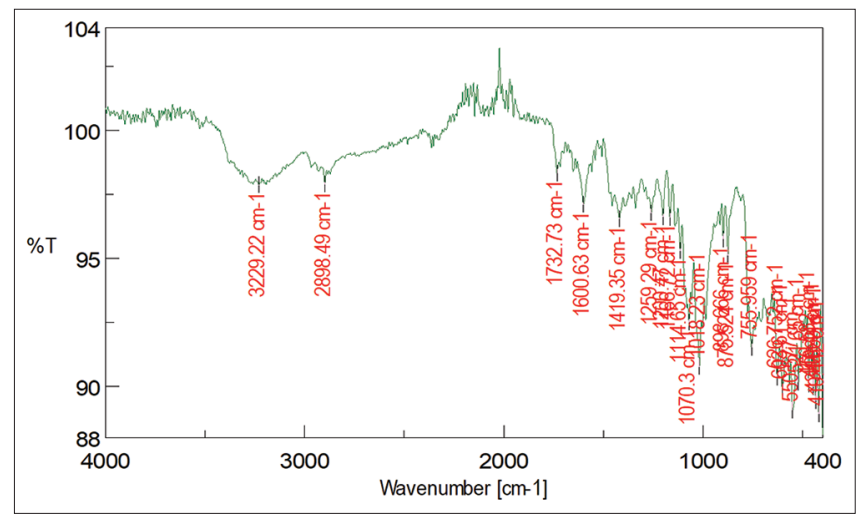

Figure 5: Fourier-transform infrared spectrum of Fs

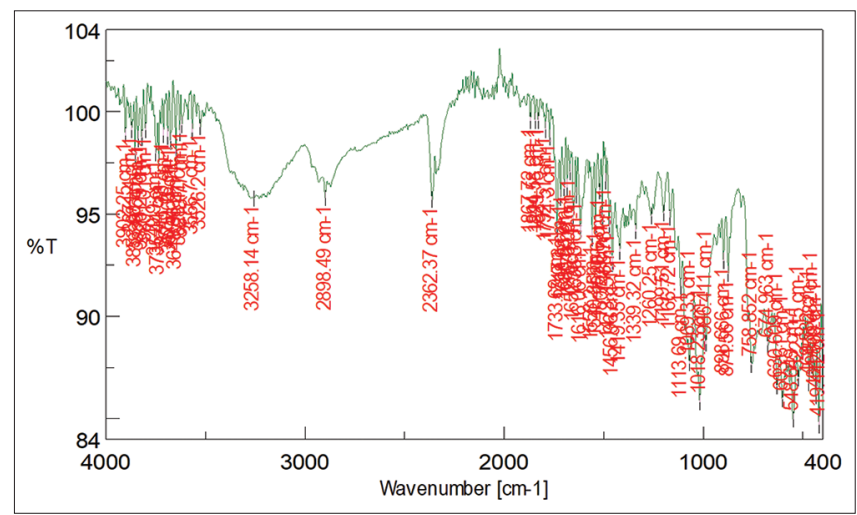

Figure 6: Fourier-transform infrared spectrum of Ft

Table 2: Weight variation test of Fc, Fs, and Ft with Fd Formula name Weight variation $(\mathrm{mg})$

FC $298.4 \pm 2.86$

Fs

$300.87 \pm 2.99$

$\mathrm{Ft}$ $300.08 \pm 2.74$

$\mathrm{Fd}$

$80.2 \pm 0.69$

\section{DISCUSSION}

\section{Pre-formulation Evaluation Parameters of Pure Valsartan Powder}

The rational purity of pure Valsartan powder confirmed by the result obtained from measurement 
Table 3: Weight content uniformity of Fc, Fs, and Ft with Fd Formula name Weight uniformity (\%) Fc $97.25 \pm 1.27$ $98.43 \pm 1.25$ $97.88 \pm 1.33$ $99.96 \pm 1.23$

Table 4: Dissolution profile of Fc, Fs, Ft, and Fd

\begin{tabular}{lcccc}
\hline $\begin{array}{l}\text { Time } \\
(\mathbf{m i n})\end{array}$ & $\begin{array}{c}\text { Percentage } \\
\text { of release of } \\
\text { Fc (\%) }\end{array}$ & $\begin{array}{c}\text { Percentage } \\
\text { of release of } \\
\text { Fs (\%) }\end{array}$ & $\begin{array}{c}\text { Percentage } \\
\text { of release of } \\
\text { Ft (\%) }\end{array}$ & $\begin{array}{c}\text { Percentage } \\
\text { of release of } \\
\text { Fd (\%) }\end{array}$ \\
\hline 5 & 35.7 & 102.88 & 104.9 & 41.20 \\
10 & 50.77 & 103.9 & 105.83 & 61.23 \\
20 & 64.6 & 97.83 & 101.33 & 77.54 \\
30 & 57.55 & 100.16 & 104.16 & 85.95 \\
45 & 45.15 & 99.83 & 103.33 & 80.23 \\
\hline
\end{tabular}

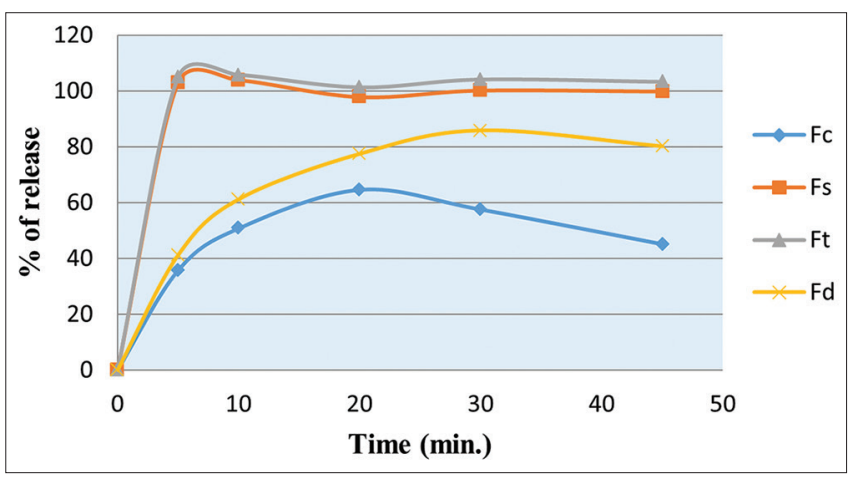

Figure 7: Dissolution profile of Fc, Fs, and Ft with Fd

of melting point and Lamda max (Khan et al., 2010; Moffat, 2011).

\section{Post-Formulation Evaluation Parameters of Valsartan Sachet}

Post-formulation evaluation parameters were performed for the prepared formulas Fc, Fs, and Ft with $\mathrm{Fd}$ as a reference.

\section{Drug-excipient Compatibility Study by FTIR Analysis} The comparison of the IR spectrum of pure drug with that of Fc, Fs, and Ft showed all the characteristic peaks of pure Valsartan and revealed that there is no obvious change in the positions of the characteristic absorption bands of groups and bonds. This clearly suggests that the drug remains in the same form even in its formulations indicating that there is no interaction between the drug and excipients were used for the preparation of Valsartan sachet (Balamuralidhara et al., 2009).

\section{Weight Variation Test}

The results of weight variation test indicated that all prepared formulas (Fc, Fs, and $\mathrm{Ft}$ ) with $\mathrm{Fd}$ were within pharmacopoeial limits (not more than two tablets differ from the average by no more than $10 \%$ and no tablets differ by more than 20\%) (Takeuchi et al., 2005). The result indicates that there were a non-significant differences $(P>0.05)$ among all formulas.

\section{Weight Content Uniformity}

The percentage of drug content uniformity of the prepared Valsartan sachet (Fc, Fs, and Ft) complies with USP criteria. It was found that no sachet from ten sachets lies out of the label claim (Balamuralidhara et al., 2009), which indicates a uniform drug distribution and a proper dose of the drug in the sachet. Therefore, the results indicate that there were a non-significant differences $(P>0.05)$ between Fc, Fs, and Ft with Fd.

\section{Dissolution Test of Fc, Fs, and Ft in Comparison with Fd}

The improvement of oral bioavailability for water in-soluble drugs remains one of the most challenging aspects of drug perfection. It has been studied a complete dissolution of drug which was obtained from solid dispersions using surface active agents such as tween 80 and SLS or selfemulsifying carriers. The vehicles acted as dispersing or emulsifying agents for the liberated drug, thus preventing the formation of any water-insoluble surface layers. Because of surface activity of the dissolved part, it was dispersed or emulsified in a finely divided state. The high surface area of a drug produced in this way could facilitate its dissolution in the dissolution medium (Faruki et al., 2013).

The oral dosage formulations containing SLS and tween 80 as a wetting agent/surfactant could improve the dissolution rate of poorly water soluble drugs like Valsartan where the drug substance and surfactants combination are used in an aqueous media. Intimate contact between drug and surfactants can occur to the lower the surface tension of the drug substance, or increase its solubility through micelle formation in the gastrointestinal contents to enhance dissolution (Alhmoud et al., 2014).

Several studies have been shown the effect of cosolvency and micellization to increase drug solubility (Alkhamis et al., 2003; Meera et al., 2010; Mbah, 2006). In addition, a series of studies on the improvement of aqueous solubility of Valsartan were done. This study tried to investigate the effect of SLS and tween 80 on the solubility of Valsartan.

The surfactants could play a key role as solubilizes and wetting agents for poorly water soluble drugs. The dissolution rate of Valsartan was increased using SLS and tween 80. Increasing wettability and spreadability of the precipitated drug caused by reducing aggregations in the readily soluble state. The vehicles with emulsifying agents enhanced liberating drugs and improved its drug solubility (Faruki et al., 2013). 
The rate and extent of dissolution of the active ingredient from any dosage form often determines the rate and extent of absorption of the drug (El-Badry and Fathy, 2006). In case of Valsartan which has low water solubility, dissolution may be the rate-limiting step in the process of drug absorption; therefore, drugs with low water solubility have been shown to be unpredictably and slowly absorbed compared with drugs of higher solubility. Oral formulation of Valsartan sachet (Fs and Ft) could be developed by increasing the water solubility and improving its efficacy.

According to USP pharmacopeia, $75 \%$ of the drug must be released from solid dosage form within $30 \mathrm{~min}$. The drug release within 30 min was $100.16 \%$ and $104.16 \%$ for Fs and Ft, respectively, which is within the acceptable limit, while for Fc only $57.55 \%$ of the drug was released. Furthermore, the results showed that after 5, 10, 20, and $45 \mathrm{~min}$ the percentage of drug release from Fs was $102.88 \%, 103.9 \%, 97.83 \%$, and $99.83 \%$, respectively, and for Ft was 104.9\%, 105.83\%, 101.33\%, and 103.33\%, respectively, whereas the percentage of drug release for $\mathrm{Fc}$ at $5,10,20$, and $45 \mathrm{~min}$ was $35.7 \%, 50.77 \%, 64.6 \%$, and $45.15 \%$, respectively. Indicating a significant differences $(P<0.05)$ in the dissolution of $\mathrm{Fs}$ and $\mathrm{Ft}$ which were faster than Fc of the prepared Valsartan sachet, while a non-significant differences $(P>0.05)$ between Fs and $\mathrm{Ft}$ with $\mathrm{Fd}$ as a reference tablets (Diovan ${ }^{\circledR} 80 \mathrm{mg}$ tablet).

This significant improvement in the dissolution profile of the prepared Valsartan sachet (Fs and Ft) refers to the content of SLS and tween 80, respectively, which are acting as solubilizing agents compared to Fc formula, while the same improvement not observed for Fc due to poor water solubility of Valsartan.

\section{CONCLUSION}

Valsartan sachet can be used as alternative dosage form after improvement of the solubility by addition of solubilizing agent such as SLS in Fs and tween 80 in Ft formula. The prepared formulas Fs and Ft provided a significant improvement in the drug release profile in comparison with $\mathrm{Fc}_{\mathrm{c}}$ and the marketed conventional Valsartan tablet (Fd). The overall results support the possibility of using the prepared formulas Fs and Ft in the preparation of Valsartan sachet as a new dosage form for the oral administration with fast dissolution time that can be used as alternative to the conventional Valsartan tablets Fd.

\section{COMPETING ITERESTS}

The authors declare that they have no competing interests.

\section{REFERENCES}

Alhmoud, H., N. E. Ibrahim, E. El-Hallous and Surfactants Solubility. 2014. Concentration and the other formulations effects on the drug release rate from a controlled release matrix. Afr. J. Pharm. Pharmacol. 8(13): 364-371.

Alkhamis, K. A., H. Allaboun and W. Y. Al-Momani. 2003. Study of the solubilization of gliclazide by aqueous micellar solutions. J. Pharm. Sci. 92(4): 839-846.

Aulton, M. E. 2000. Pharmaceutics the Science of Dosage form Design. $3^{\text {rd }}$ ed. Churchill Livingstone, New York. p.25-27.

Balamuralidhara, V., S. A. Sreenivas, H. V. Gangadhara and T. M. Pramodkumar. 2009. Investigation on the effect of different disintegrants on the orodispersible tablets of rabeprazole. Asian J. Sci. Res. 4: 12-19.

Bharti, V. P., V. R. Attal, A. V. Munde, A. S. Birajdar and S. Bais. 2015. Strategies to enhance solubility and dissolution of a poorly water soluble drug. J. Innov. Pharm. Biol. Sci. 2(4): 482-494.

Dalmora, M. E., S. L. Dalmora and A. G. Oliveira. 2001. Inclusion complex of piroxicam with b-cyclodextrin and incorporation in cationic microemulsion. In vitro drug release and in vivo topical anti-inflammatory effect. Int. J. Pharm. 222: 45-55.

El-Badry, M. and M. Fathy. 2006. Enhancement of dissolution and permeation rates of meloxicam by formation of its freeze dried solid dispersion in polyvinyl pyrrolidine K-30. Drug Dev. Ind. Pharm. 32(2): 141-142.

Faruki, Z. M., Rishikesh, E. Razzaque and M. A. Bhuiyan. 2013. Improvement of solubility of badly water soluble drug (ibuprofen) by using surfactants and carriers. Int. J. Pharm. Sci. Res. 4(4): 1569-1574.

Flesch, G., P. Muller and P. Lloyd. 1997. Absolute bioavailability and pharmacokinetics of valsartan, an angiotensin II receptor antagonist. Eur. J. Clin. Pharmacol. 52(2): 115-120.

Gilman, A. G., T. W. Rall., A. S. Nies and P. Taylor. 1990. The Pharmacological Basis of Therapeutics. $8^{\text {th }}$ ed., Vol. 2. McGrawHill, New York. p50-64.

Kawabataa, Y., K. Wadab, M. Nakatanib and S. Yamadaa. 2011. Formulation design for poorly water-soluble drugs based on biopharmaceutics classification system: Basic approaches and practical applications. Int. J. Pharm. 420(1): 1-10.

Khan, F., R. T. Lohiya and M. J. Umekar. 2010. Development of UV spectrophotometric method for the simultaneous estimation of meloxicam and paracetamol in tablet by simultaneous equation, absorbance ratio and absorbance correction method. Int. J. ChemTech. Res. 2(3): 1586-1591.

Lawrence, M. J. and G. D. Rees. 2000. Microemulsions based media as novel drug delivery systems. Adv. Drug Deliv. Rev. 45(1): 89-121.

Lobenberg, R. and G. L. Amidon. 2000. Modern bioavailability, bioequivalence and biopharmaceutics classification system: New scientific approaches to international regulatory standards. Eur. J. Pharm. Biopharm. 50(1): 3-12.

Mbah, C. J. 2006. Solubilization of valsartan by aqueous glycerol, polyethylene glycol and micellar solutions. Pharm. J. 61: 322-324.

Meera, C. A. B. Singh, Sayyad and S. D. Sawant. 2010. Review on various techniques of solubility enhancement of poorly soluble drugs with special emphasis on solid dispersion. J. Pharm. Res. 3(10): 2494-2501.

Moffat, A. C. 2011. Clarke's Analysis of Drugs and Poisons. Pharmaceutcal Press, London, United Kingdom.

Nayak, B. S., S. R. Mishra and H. Roy. 2018. Valsartan fast dissolving tablets: Formulation and in vitro characterization. J. Chem. Pharm. Res. 10(3): 182-189. 
Pedersen, B. L., A. Mullertz, H. Brondsted and H. C. Kristenson. 2000. A comparision of the solubility of danazol in human and simulated gastrointestinal fluids. Pharm. Res. 17(7): 891-894.

Pradhan, K. K. and U. S. Mishra. 2015. Development and validation of a stability indicating RP-HPLC method for the determination of valsartan. Int. J. Pharm. Pharm. Sci. 7(8): 57-61.

Rabasco, A. M., J. M. Ginesh, M. F. Arevalo and M. A. Holgado. 2002. Dissolution rate of diazepam from polyethylene glycol 6000 solid dispersions. Int. J. Pharm. 67: 201-206.

Rong, L. 2008. Water Insoluble Drug-formulation. $2^{\text {nd }}$ ed. CRC Press, New York. p1-4.

Rowe, R. C., P. J. Sheskey and S. C. Owen. 2009. Handbook of Pharmaceutical Ecipients. $6^{\text {th }}$ ed. Pharmaceutical Press, American Pharmacists Association, London, United Kingdom.

Takeuchi, H., S. Tanimura, S. Y. Nagira, H. Yamamoto and Y. Kawashina. 2005. Tableting of solid dispersion particles consisiting of indomathacin and porous silica particles. Chem. Pharm. Bull. 53(5): 487-491.

Usha, B. S., Y. I. Muzib, D. Bhikshapathi and R. Sravani. 2015. Enhancement of solubility and oral bioavailability of poorly soluble drug valsartan by novel solid self emulsifying drug delivery system. Int. J. Drug Deliv. 7: 13-26.

USP-30 NF-25. 2007. United State Pharmacopoeial Convention. Electronic Version. 